\title{
Riesz Means of Dirichlet Eigenvalues for the Sub-Laplace Operator on the Engel Group
}

\author{
Jingjing Xue \\ Department of Applied Mathematics, Northwestern Polytechnical University, Xi'an, China \\ Email: xuejingjingsx@163.com
}

Received September 27, 2013; revised October 27, 2013; accepted November 5, 2013

Copyright (C) 2013 Jingjing Xue. This is an open access article distributed under the Creative Commons Attribution License, which permits unrestricted use, distribution, and reproduction in any medium, provided the original work is properly cited.

\section{ABSTRACT}

In this paper, we are concerned with the Riesz means of Dirichlet eigenvalues for the sub-Laplace operator on the Engel group and deriver different inequalities for Riesz means. The Weyl-type estimates for means of eigenvalues are given.

Keywords: Engel Group; Sub-Laplace Operator; Eigenvalues; Riesz Mean

\section{Introduction}

The Engel group $G$ is a Carnot group of step $r=3$ (see [1]), its Lie algebra is generated by the left-invariant vector fields

$$
\begin{aligned}
& X_{1}=\frac{\partial}{\partial x_{1}}-\frac{x_{2}}{2} \frac{\partial}{\partial x_{3}}+\left(\frac{-x_{1} X_{2}}{12}-\frac{x_{3}}{2}\right) \frac{\partial}{\partial w}, \\
& X_{2}=\frac{\partial}{\partial x_{2}}+\frac{x_{1}}{2} \frac{\partial}{\partial x_{3}}+\frac{x_{1}^{2}}{12} \frac{\partial}{\partial w}, \\
& X_{3}=\frac{\partial}{\partial x_{3}}+\frac{x_{1}}{2} \frac{\partial}{\partial w} \\
& X_{4}=\frac{\partial}{\partial w},
\end{aligned}
$$

where $P=\left(x_{1}, x_{2}, x_{3}, w\right)$ is a point of $G$. It is easy to see that

$$
\begin{aligned}
& {\left[X_{1}, X_{2}\right]=X_{3},\left[X_{1}, X_{3}\right]=X_{4},\left[X_{2}, X_{3}\right]=0,} \\
& {\left[X_{1}, X_{4}\right]=\left[X_{2}, X_{4}\right]=0,}
\end{aligned}
$$

and $\left[X_{3}, X_{4}\right]=0$. So the Lie algebra of $G$ is

$$
g=V_{1} \oplus V_{2} \oplus V_{3},
$$

where $V_{1}=\operatorname{span}\left\{X_{1}, X_{2}\right\}, \quad V_{2}=\operatorname{span}\left\{X_{3}\right\}$ and $V_{3}=\operatorname{span}\left\{X_{4}\right\}$. The sub-Laplace operator on $G$ is of the form $\Delta_{E}=X_{1}^{2}+X_{2}^{2}$.

In the paper, we investigate the Riesz means of the Dirichlet problem

$$
\begin{cases}-\Delta_{E} u=\lambda u, & \text { in } \Omega, \\ u=0, & \text { on } \partial \Omega .\end{cases}
$$

in the Engel group $G$. Here $\Omega$ is a bounded and noncharacteristics domain in $G$, with smooth boundary $\partial \Omega$. The existence of eigenvalues for (1.1) is from [2]. Let us by $R_{\sigma}(z)$ denote the Riesz means of order $\sigma$ of the sequence $\left\{\lambda_{k}\right\}$ of eigenvalues of (1.1).

The Riesz means of Dirichlet eigenvalues for the Laplace operator in the Euclidean space have been extensively studied(see [3-5]). In recent years, E. M. Harrell II and L. Hermi in [6] treated the Riesz means $R_{\sigma}(z)$ of order $\sigma$ of $\left\{\lambda_{k}\right\}$ on the bounded domain $\Omega \subset R^{d}$ and pointed out that: for $0<\sigma \leq 2$ and $z \geq \lambda_{1}$,

$$
\begin{aligned}
& R_{\sigma-1}(z) \geq\left(1+\frac{d}{4}\right) \frac{1}{z} R_{\sigma}(z) \\
& \text { and } R_{\sigma}^{\prime}(z) \geq\left(1+\frac{d}{4}\right) \frac{\sigma}{z} R_{\sigma}(z),
\end{aligned}
$$

and $\frac{R_{\sigma}(z)}{z+\frac{d \sigma}{4}}$ is a nondecreasing function of $z$; for $2<\sigma<+\infty$ and $z \geq \lambda_{1}$,

$$
\begin{aligned}
& R_{\sigma-1}(z) \geq\left(1+\frac{d}{2 \sigma}\right) \frac{1}{z} R_{\sigma}(z) \\
& \text { and } R_{\sigma}^{\prime}(z) \geq\left(\sigma+\frac{d}{2}\right) \frac{1}{z} R_{\sigma}(z),
\end{aligned}
$$


and $\frac{R_{\sigma}(z)}{z+\frac{d}{2}}$ is a nondecreasing function of $z$, and then the Weyl-type estimates of means of eigenvalues is derived.

Jia et al. in [7] extended (1.2), (1.3) to the Heisenberg group.

The main results of this paper are the following.

Theorem 1.1 For $0<\sigma \leq 2$ and $z \geq \lambda_{1}$, we have

$$
\begin{aligned}
& R_{\sigma-1}(z) \geq \frac{3}{2 z} R_{\sigma}(z), \\
& R_{\sigma}^{\prime}(z) \geq \frac{3}{2} \frac{\sigma}{z} R_{\sigma}(z),
\end{aligned}
$$

and $\frac{R_{\sigma}(z)}{z^{\frac{3 \sigma}{2}}}$ is a nondecreasing function of $z$; for $2<\sigma<+\infty$ and $z \geq \lambda_{1}$, we have

$$
\begin{aligned}
& R_{\sigma-1}(z) \geq\left(1+\frac{1}{\sigma}\right) \frac{1}{z} R_{\sigma}(z), \\
& R_{\sigma}^{\prime}(z) \geq(\sigma+1) \frac{1}{z} R_{\sigma}(z),
\end{aligned}
$$

and $\frac{R_{\sigma}(z)}{z^{\sigma+1}}$ is a nondecreasing function of $z$.

Theorem 1.2 Suppose that $z \geq 3 \overline{\lambda_{j}}$, then

$$
R_{2}(z) \geq \frac{4 j z^{3}}{27 \bar{\lambda}_{j}}
$$

and therefore

$$
\begin{gathered}
R_{1}(z) \geq \frac{2 j z^{2}}{9 \overline{\lambda_{j}}}, \\
N(z)=R_{0}(z) \geq \frac{j z}{3 \bar{\lambda}_{j}},
\end{gathered}
$$

Moreover, for all $k \geq j \geq 1$, we have the upper bound

$$
\lambda_{k+1} \leq \frac{3 k}{j} \bar{\lambda}_{j} \text {. }
$$

Theorem 1.3 For $k>\frac{4 j}{3}$, we have

$$
\frac{\overline{\lambda_{k}}}{\overline{\lambda_{j}}} \leq \frac{9 k}{8 j} .
$$

Authors in [6] combined the Weyl-type estimates of means of eigenvalues established in [6] and the result in [8] to obtain the Weyl-type estimates of eigenvalues. But it is not easy to extend the result in [8] to the Engel group. The Weyl-type estimates of eigenvalues for (1.1) still are open questions.

This paper is arranged as follows. In Section 2 the definition of Riesz means and Lemmas are described; Section 3 is devoted to the proof of Theorem 1.1. The proof of Theorem 1.2 is appeared in Section 4. In Section 5 the proof of Theorem 1.3 is given.

\section{Preliminaries}

Definition 2.1 For an increasing sequence $\left\{\lambda_{k}\right\}_{k=1}^{\infty}$ of real numbers and $z \geq 0$, the Riesz means $R_{\sigma}(z)$ of order $\sigma>0$ of $\left\{\lambda_{k}\right\}$ is defined by

$$
R_{\sigma}(z)=\sum_{k=1}^{\infty}\left(z-\lambda_{k}\right)_{+}^{\sigma},
$$

where $\left(z-\lambda_{k}\right)_{+}=\max \left\{0, z-\lambda_{k}\right\}$ is the ramp function.

Clearly,

$$
R_{\sigma}^{\prime}(z)=\sigma R_{\sigma-1}(z) .
$$

Similarly to Theorem 1 of [9], we immediately have

Lemma 2.2 Denoting the $L^{2}$-normalized eigenfunctions of (1.1) by $\left\{u_{j}\right\}$, let

$$
T_{\alpha j m}=\left|\left(X_{\alpha} u_{j}, u_{m}\right)\right|^{2}
$$

for $\alpha=1,2 ; j, m=1,2, \cdots$. Then for each fixed $\alpha$, we have

$$
\begin{aligned}
& R_{\sigma}(z)= \\
& 2 \sum_{j, m: \lambda_{j} \neq \lambda_{m}} \frac{\left(z-\lambda_{j}\right)_{+}^{\sigma}-\left(z-\lambda_{m}\right)_{+}^{\sigma}}{\lambda_{m}-\lambda_{j}} T_{\alpha j m} \\
& +4 \sum_{j, q: \lambda_{j} \leq z<\lambda_{q}} \frac{\left(z-\lambda_{j}\right)^{\sigma}}{\lambda_{q}-\lambda_{j}} T_{\alpha j q} .
\end{aligned}
$$

Lemma 2.3 ([10]) Let $0<x<y$ and $\sigma \geq 0$, then

$$
\frac{y^{\sigma}-x^{\sigma}}{y-x} \leq C_{\sigma}\left(y^{\sigma-1}+x^{\sigma-1}\right) \text {, }
$$

where

$$
C_{\sigma}= \begin{cases}\frac{\sigma}{2}, & 0 \leq \sigma<1, \\ 1, & 1 \leq \sigma \leq 2, \\ \frac{\sigma}{2}, & 2 \leq \sigma<+\infty .\end{cases}
$$

\section{The Proof of Theorem 1.1}

In this section, we prove Theorem 1.1 and two corollaries.

Proof. Let us use (2.2) and denote the first term on the right-hand side of (2.2) by $G(\sigma, z, \alpha)$. Applying Lemma 2.3 it follows 


$$
\begin{aligned}
& G(\sigma, z, \alpha)=2 \sum_{j, m: \lambda_{j} \neq \lambda_{m}} \frac{\left(z-\lambda_{j}\right)_{+}^{\sigma}-\left(z-\lambda_{m}\right)_{+}^{\sigma}}{\lambda_{m}-\lambda_{j}} T_{\alpha j m} \\
& =2 \sum_{j, m: \lambda_{j} \leq z, \lambda_{m} \leq z, \lambda_{j} \neq \lambda_{m}} \frac{\left(z-\lambda_{j}\right)^{\sigma}-\left(z-\lambda_{m}\right)^{\sigma}}{\lambda_{m}-\lambda_{j}} T_{\alpha j m} \\
& \leq 2 C_{\sigma} \sum_{j, m: \lambda_{j} \leq z, \lambda_{m} \leq z, \lambda_{j} \neq \lambda_{m}}\left[\left(z-\lambda_{j}\right)^{\sigma-1}+\left(z-\lambda_{m}\right)^{\sigma-1}\right] T_{\alpha j m} \\
& =4 C_{\sigma} \sum_{j, m: \lambda_{j} \leq z, \lambda_{m} \leq z}\left(z-\lambda_{j}\right)_{+}^{\sigma-1} T_{\alpha j m} \\
& =4 C_{\sigma} \sum_{j: \lambda_{j} \leq z, a l l m}\left(z-\lambda_{j}\right)_{+}^{\sigma-1} T_{\alpha j m} \\
& -4 C_{\sigma} \sum_{j, q: \lambda_{j} \leq z<\lambda_{q}}\left(z-\lambda_{j}\right)_{+}^{\sigma-1} T_{\alpha j q},
\end{aligned}
$$

here we used the symmetry on $j$ and $m$ in the last step.

Putting the above estimate into (2.2), we have

$$
\begin{aligned}
& R_{\sigma}(z) \leq 4 C_{\sigma} \sum_{j: \lambda_{j} \leq z, \text { all } m}\left(z-\lambda_{j}\right)_{+}^{\sigma-1} T_{\alpha j m} \\
& -4 C_{\sigma} \sum_{j, q: \lambda_{j} \leq z<\lambda_{q}}\left(z-\lambda_{j}\right)_{+}^{\sigma-1} T_{\alpha j q} \\
& +4 \sum_{j, q: \lambda_{j} \leq z<\lambda_{q}} \frac{\left(z-\lambda_{j}\right)^{\sigma}}{\lambda_{q}-\lambda_{j}} T_{\alpha j q} \\
& =4 C_{\sigma} \sum_{j: \lambda_{j} \leq z, a \text { all } m}\left(z-\lambda_{j}\right)_{+}^{\sigma-1} T_{\alpha j m} \\
& +4 \sum_{j, q: \lambda_{j} \leq z<\lambda_{q}} T_{\alpha j q}\left(z-\lambda_{j}\right)^{\sigma-1}\left(\frac{z-\lambda_{j}-C_{\sigma}\left(\lambda_{q}-\lambda_{j}\right)}{\lambda_{q}-\lambda_{j}}\right) \\
& =4 C_{\sigma} \sum_{j: \lambda_{j} \leq z, \text { all } m}\left(z-\lambda_{j}\right)_{+}^{\sigma-1} T_{\alpha j m}+4 H(\sigma, z, \alpha),
\end{aligned}
$$

where we denote

$$
\begin{aligned}
& H(\sigma, z, \alpha) \\
& =\sum_{j, q: \lambda_{j} \leq z<\lambda_{q}} T_{\alpha j q}\left(z-\lambda_{j}\right)^{\sigma-1}\left(\frac{z-\lambda_{j}-C_{\sigma}\left(\lambda_{q}-\lambda_{j}\right)}{\lambda_{q}-\lambda_{j}}\right) .
\end{aligned}
$$

Since $\left\{u_{m}\right\}$ is a complete orthonormal set, it follows

$$
\sum_{m=1}^{\infty} T_{\alpha j m}=\left\|X_{\alpha} u_{j}\right\|^{2}
$$

and

$$
\begin{aligned}
\sum_{\alpha=1 m=1}^{2} \sum_{\alpha j m}^{\infty} T_{\alpha j} & =\left\|X_{1} u_{j}\right\|^{2}+\left\|X_{2} u_{j}\right\|^{2}=\int\left|\nabla_{E} u_{j}\right|^{2} \\
& =\int\left(\nabla_{E} u_{j}\right) \cdot\left(\nabla_{E} u_{j}\right)=-\int u_{j} \cdot \Delta_{E} u_{j} \\
& =\int \lambda_{j} u_{j}^{2}=\lambda_{j} .
\end{aligned}
$$

Returning to (3.1) with them, it yields

$$
2 R_{\sigma}(z) \leq 4 C_{\sigma} \sum_{j}\left(z-\lambda_{j}\right)_{+}^{\sigma-1} \lambda_{j}+4 \sum_{\alpha=1}^{2} H(\sigma, z, \alpha) .
$$

Since

$$
\sum_{j}\left(z-\lambda_{j}\right)_{+}^{\sigma-1} \lambda_{j}=z R_{\sigma-1}(z)-R_{\sigma}(z)
$$

we have

$$
2 R_{\sigma}(z) \leq 4 C_{\sigma}\left(z R_{\sigma-1}(z)-R_{\sigma}(z)\right)+4 \sum_{\alpha=1}^{2} H(\sigma, z, \alpha),
$$

namely,

$$
\left(1+2 C_{\sigma}\right) R_{\sigma}(z)-2 z C_{\sigma} R_{\sigma-1}(z) \leq 2 \sum_{\alpha=1}^{2} H(\sigma, z, \alpha) .
$$

We consider three cases: 1) $1 \leq \sigma \leq 2$; 2) $0<\sigma<1$ and 3) $\sigma>2$.

1) $1 \leq \sigma \leq 2$. In this case, it sees $C_{\sigma}=1$ and

$$
\frac{z-\lambda_{j}-C_{\sigma}\left(\lambda_{q}-\lambda_{j}\right)}{\lambda_{q}-\lambda_{j}}=\frac{z-\lambda_{q}}{\lambda_{q}-\lambda_{j}} .
$$

Since $\lambda_{q}>z$, it follows

$$
\frac{z-\lambda_{j}-C_{\sigma}\left(\lambda_{q}-\lambda_{j}\right)}{\lambda_{q}-\lambda_{j}}<0,
$$

and therefore

$$
H(\sigma, z, \alpha)<0 .
$$

Substituting this into (3.4), we obtain

$$
\left(1+2 C_{\sigma}\right) R_{\sigma}(z)-2 z C_{\sigma} R_{\sigma-1}(z) \leq 0
$$

and

$$
R_{\sigma-1}(z) \geq \frac{3}{2 z} R_{\sigma}(z) .
$$

Now (1.4) is proved.

Using (2.1), we have

$$
\frac{1}{\sigma} R_{\sigma}^{\prime}(z) \geq \frac{3}{2 z} R_{\sigma}(z),
$$

and (1.5) is proved.

Since

$$
\left(\frac{R_{\sigma}(z)}{z^{\frac{3 \sigma}{2}}}\right)^{\prime}=\frac{R_{\sigma}^{\prime}(z) z^{\frac{3 \sigma}{2}}-R_{\sigma}(z) \frac{3 \sigma}{2} z^{\frac{3 \sigma}{2}-1}}{z^{3 \sigma}}
$$

$$
=\frac{z^{\frac{3 \sigma}{2}-1}\left[z R_{\sigma}^{\prime}(z)-\frac{3 \sigma}{2} R_{\sigma}(z)\right]}{z^{3 \sigma}} \geq 0,
$$

it follows that $\frac{R_{\sigma}(z)}{z^{\frac{3 \sigma}{2}}}$ is a nondecreasing function of $z$. 
2) $0<\sigma<1$. Now $C_{\sigma}=\frac{\sigma}{2} \in\left(0, \frac{1}{2}\right)$, so $1-C_{\sigma}>0$ and

$$
\begin{aligned}
\frac{z-\lambda_{j}-C_{\sigma}\left(\lambda_{q}-\lambda_{j}\right)}{\lambda_{q}-\lambda_{j}} & <\frac{\lambda_{q}-\lambda_{j}-C_{\sigma}\left(\lambda_{q}-\lambda_{j}\right)}{\lambda_{q}-\lambda_{j}} \\
& =1-C_{\sigma} .
\end{aligned}
$$

Then

$$
\begin{aligned}
H(\sigma, z, \alpha) & \leq\left(1-C_{\sigma}\right) \sum_{j, q: \lambda_{q}>z} T_{\alpha j q}\left(z-\lambda_{j}\right)_{+}^{\sigma-1} \\
& \leq\left(1-C_{\sigma}\right) \sum_{j, q} T_{\alpha j q}\left(z-\lambda_{j}\right)_{+}^{\sigma-1}
\end{aligned}
$$

and

$$
\begin{aligned}
\sum_{\alpha=1}^{2} H(\sigma, z, \alpha) & \leq\left(1-C_{\sigma}\right) \sum_{j}\left(z-\lambda_{j}\right)_{+}^{\sigma-1} \lambda_{j} \\
& =\left(1-C_{\sigma}\right)\left(z R_{\sigma-1}(z)-R_{\sigma}(z)\right) .
\end{aligned}
$$

Substituting this into (3.4), we obtain

$$
\begin{aligned}
& \left(1+2 C_{\sigma}\right) R_{\sigma}(z)-2 z C_{\sigma} R_{\sigma-1}(z) \\
& \leq\left(2-2 C_{\sigma}\right)\left[z R_{\sigma-1}(z)-R_{\sigma}(z)\right],
\end{aligned}
$$

namely,

$$
3 R_{\sigma}(z) \leq 2 z R_{\sigma-1}(z),
$$

and (1.4) is proved.

The remainders are discussed similarly to 1 ).

3) $\sigma>2$. In this case $C_{\sigma}=\frac{\sigma}{2}>1$, so $1-C_{\sigma}<0$ and

$$
H(\sigma, z, \alpha) \leq\left(1-C_{\sigma}\right) \sum_{j, q: \lambda_{q}>z} T_{\alpha j q}\left(z-\lambda_{j}\right)_{+}^{\sigma-1}<0 .
$$

Substituting this into (3.4), we have

$$
\left(1+2 C_{\sigma}\right) R_{\sigma}(z) \leq 2 z C_{\sigma} R_{\sigma-1}(z)
$$

and (1.6) is proved.

Noting (2.1), it implies

$$
\frac{1}{\sigma} R_{\sigma}^{\prime}(z) \geq\left(1+\frac{1}{\sigma}\right) \frac{1}{z} R_{\sigma}(z)
$$

and (1.7) is proved.

Similarly,

$$
\begin{aligned}
\left(\frac{R_{\sigma}(z)}{z^{\sigma+1}}\right)^{\prime} & =\frac{R_{\sigma}^{\prime}(z) z^{\sigma+1}-R_{\sigma}(z)(\sigma+1) z^{\sigma}}{z^{2(\sigma+1)}} \\
& =\frac{z^{\sigma}\left[z R_{\sigma}^{\prime}(z)-(\sigma+1) R_{\sigma}(z)\right]}{z^{2(\sigma+1)}} \geq 0,
\end{aligned}
$$

thus $\frac{R_{\sigma}(z)}{z^{\sigma+1}}$ is a nondecreasing function of $z$.
Corollary 3.1 For all $\sigma \geq 2$ and $z \geq(1+\sigma) \lambda_{1}$,

$$
\sigma^{\sigma} \lambda_{1}^{-1}\left(\frac{z}{1+\sigma}\right)^{1+\sigma} \leq R_{\sigma}(z) \leq L_{\sigma, 2}^{c l}|\Omega| z^{\sigma+1},
$$

where $L_{\sigma, 2}^{c l}=\frac{\Gamma(\sigma+1)}{4 \pi \Gamma(\sigma+2)}$.

Proof. 1) Noting $R_{\sigma}\left(z_{0}\right)=\sum_{k}\left(z_{0}-\lambda_{k}\right)_{+}^{\sigma} \geq\left(z_{0}-\lambda_{1}\right)_{+}^{\sigma}$, for any $z_{0}>\lambda_{1}$, it follows from Theorem 1.1 that for all $z \geq z_{0}$

$$
\frac{R_{\sigma}(z)}{z^{\sigma+1}} \geq \frac{R_{\sigma}\left(z_{0}\right)}{z_{0}^{\sigma+1}} \geq \frac{\left(z_{0}-\lambda_{1}\right)_{+}^{\sigma}}{z_{0}^{\sigma+1}} .
$$

So

$$
R_{\sigma}(z) \geq\left(z_{0}-\lambda_{1}\right)_{+}^{\sigma}\left(\frac{z}{z_{0}}\right)^{\sigma+1} .
$$

Since (3.7) holds for arbitrary $z_{0}>\lambda_{1}$, it yields

$$
R_{\sigma}(z) \geq \max _{z_{0}>\lambda_{1}}\left[\left(z_{0}-\lambda_{1}\right)_{+}^{\sigma}\left(\frac{z}{z_{0}}\right)^{\sigma+1}\right] .
$$

Due to

$$
\begin{aligned}
& {\left[\left(z_{0}-\lambda_{1}\right)_{+}^{\sigma}\left(\frac{1}{z_{0}}\right)^{\sigma+1}\right]^{\prime}} \\
& =\frac{\sigma\left(z_{0}-\lambda_{1}\right)_{+}^{\sigma-1} z_{0}^{(\sigma+1)}-(\sigma+1)\left(z_{0}-\lambda_{1}\right)_{+}^{\sigma} z_{0}^{\sigma}}{z_{0}^{2(\sigma+1)}} \\
& =\frac{\left(z_{0}-\lambda_{1}\right)_{+}^{\sigma-1}\left[\sigma z_{0}-(\sigma+1)\left(z_{0}-\lambda_{1}\right)_{+}\right]}{z_{0}^{\sigma+2}},
\end{aligned}
$$

we see that when $z_{0}=(\sigma+1) \lambda_{1}$, it gets

$$
\max _{z_{0}>\lambda_{1}}\left[\left(z_{0}-\lambda_{1}\right)_{+}^{\sigma}\left(\frac{z}{z_{0}}\right)^{\sigma+1}\right]=\sigma^{\sigma} \lambda_{1}^{-1}\left(\frac{z}{1+\sigma}\right)^{1+\sigma} .
$$

For $z \geq z_{0}=(\sigma+1) \lambda_{1}$, we have

$$
R_{\sigma}(z) \geq \sigma^{\sigma} \lambda_{1}^{-1}\left(\frac{z}{1+\sigma}\right)^{1+\sigma}
$$

and the inequality in the left-hand side of (3.6) is valid.

2) By the Berezin-Lieb inequality (see [11]), we have

$$
\frac{R_{\sigma}(z)}{z^{\sigma+1}} \rightarrow L_{\sigma, 2}^{c l}|\Omega|, z \rightarrow \infty .
$$

Notice that $\frac{R_{\sigma}(z)}{z^{\sigma+1}}$ is nondecreasing to $z$, it follows

$$
\frac{R_{\sigma}(z)}{z^{\sigma+1}} \leq L_{\sigma, 2}^{c l}|\Omega|
$$


and the inequality in the right-hand side of (3.6) is proved.

Corollary 3.2 1) For $1 \leq \sigma \leq 2$ and $z \geq(\sigma+2) \lambda_{1}$,

$$
R_{\sigma}(z) \geq \frac{(\sigma+1)^{\sigma}}{(\sigma+2)^{\sigma+1}} \lambda_{1}^{-1} z^{\sigma+1}
$$

2) For $0 \leq \sigma<1$ and $z \geq(\sigma+3) \lambda_{1}$,

$$
R_{\sigma}(z) \geq \frac{3(\sigma+2)^{\sigma+1}}{2(\sigma+3)^{\sigma+2}} \lambda_{1}^{-1} z^{\sigma+1} .
$$

Proof. 1) By Corollary 3.1 we know that for $1 \leq \sigma \leq 2$ and $z \geq(\sigma+2) \lambda_{1}$, it holds

$$
R_{\sigma+1}(z) \geq(\sigma+1)^{\sigma+1} \lambda_{1}^{-1}\left(\frac{z}{\sigma+2}\right)^{\sigma+2}
$$

Using Theorem 1.1, we have

$$
R_{\sigma}(z) \geq\left(1+\frac{1}{\sigma+1}\right) \frac{1}{z} R_{\sigma+1}(z), \text { for } 1 \leq \sigma \leq 2 .
$$

Combining (3.10) and (3.11), it follows

$$
\begin{aligned}
R_{\sigma}(z) & \geq\left(1+\frac{1}{\sigma+1}\right) \frac{1}{z}(\sigma+1)^{\sigma+1} \lambda_{1}^{-1}\left(\frac{z}{\sigma+2}\right)^{\sigma+2} \\
& =\frac{(\sigma+1)^{\sigma}}{(\sigma+2)^{\sigma+1}} \lambda_{1}^{-1} z^{\sigma+1}
\end{aligned}
$$

and (3.8) is proved.

2) By Corollary 3.1, it shows that for $0 \leq \sigma<1$ and $z \geq(\sigma+3) \lambda_{1}$, it holds

$$
R_{\sigma+2}(z) \geq(\sigma+2)^{\sigma+2} \lambda_{1}^{-1}\left(\frac{z}{\sigma+3}\right)^{\sigma+3} .
$$

From Theorem 1.1, we see that for $0 \leq \sigma<1$,

$$
R_{\sigma}(z) \geq \frac{3}{2 z} R_{\sigma+1}(z) \geq \frac{9}{4 z^{2}} R_{\sigma+2}(z) .
$$

In the light of (3.12) and (3.13), it obtains

$$
\begin{aligned}
R_{\sigma}(z) & \geq \frac{9}{4 z^{2}} R_{\sigma+2}(z) \geq \frac{9}{4 z^{2}}(\sigma+2)^{\sigma+2} \lambda_{1}^{-1}\left(\frac{z}{\sigma+3}\right)^{\sigma+3} \\
& =\frac{3(\sigma+2)}{2(\sigma+3)} \cdot \frac{3(\sigma+2)^{\sigma+1}}{2(\sigma+3)^{\sigma+2}} \lambda_{1}^{-1} z^{\sigma+1} .
\end{aligned}
$$

Noting that $\frac{3(\sigma+2)}{2(\sigma+3)}=\frac{3}{2}\left(1-\frac{1}{\sigma+3}\right) \geq 1$, for $0 \leq \sigma<1$, we have

$$
R_{\sigma}(z) \geq \frac{3(\sigma+2)^{\sigma+1}}{2(\sigma+3)^{\sigma+2}} \lambda_{1}^{-1} z^{\sigma+1}
$$

and (3.9) is proved.

Remark 3.3 Specially, we have

$$
\begin{aligned}
& R_{1}(z) \geq \frac{3}{2 z} R_{2}(z) \geq \frac{2}{9} \lambda_{1}^{-1} z^{2}, \\
& N(z)=R_{0}(z) \geq \frac{3}{2 z} R_{1}(z) \geq \frac{9}{4 z^{2}} R_{2}(z) \geq \frac{z}{3 \lambda_{1}} .
\end{aligned}
$$

\section{Proof of Theorem 1.2}

Denote

$$
\bar{\lambda}_{j}=\frac{1}{j} \sum_{l \leq j} \lambda_{l} \quad \text { and } \quad \bar{\lambda}_{j}^{2}=\frac{1}{j} \sum_{l \leq j} \lambda_{l}^{2},
$$

and let ind $(z)$ be the greatest integer $i$ such that $\lambda_{i} \leq z$.

Let ind $(z)=i$, it implies that $\lambda_{i} \leq z$ and $\lambda_{i+1}>z$, so

$$
\begin{aligned}
& R_{2}(z)=\sum_{k}\left(z-\lambda_{k}\right)_{+}^{2} \\
& =\left(z-\lambda_{1}\right)^{2}+\left(z-\lambda_{2}\right)^{2}+\cdots+\left(z-\lambda_{i}\right)^{2} \\
& =i z^{2}-2 z\left(\lambda_{1}+\lambda_{2}+\cdots+\lambda_{i}\right)+\left(\lambda_{1}^{2}+\lambda_{2}^{2}+\cdots+\lambda_{i}^{2}\right) \\
& =i z^{2}-2 i z \frac{\lambda_{1}+\lambda_{2}+\cdots+\lambda_{i}}{i}+i \frac{\lambda_{1}^{2}+\lambda_{2}^{2}+\cdots+\lambda_{i}^{2}}{i} \\
& =i\left(z^{2}-2 z \overline{\lambda_{i}}+\overline{\lambda_{i}^{2}}\right) \\
& =i n d(z)\left(z^{2}-2 z \overline{\lambda_{\text {ind }(z)}}+\overline{\lambda_{\text {ind }(z)}^{2}}\right) .
\end{aligned}
$$

For any integer $j$ and $z \geq \lambda_{j}$, it implies ind $(z) \geq j$, and

$$
R_{2}(z) \geq Q(z, j):=j\left(z^{2}-2 z \overline{\lambda_{j}}+\overline{\lambda_{j}^{2}}\right)
$$

Using Theorem 1.1, we have that for $z \geq z_{j} \geq \lambda_{j}$,

$$
\frac{R_{2}(z)}{z^{3}} \geq \frac{Q\left(z_{j}, j\right)}{z_{j}^{3}}
$$

or

$$
R_{2}(z) \geq Q\left(z_{j}, j\right)\left(\frac{z}{z_{j}}\right)^{3} .
$$

By the Cauchy-Schwarz inequality, it follows

$$
{\overline{\lambda_{j}}}^{2} \leq \overline{\lambda_{j}^{2}}
$$

and

$$
\begin{aligned}
Q\left(z_{j}, j\right) & =j\left(z^{2}-2 z \overline{\lambda_{j}}+\overline{\lambda_{j}^{2}}\right) \\
& =j\left(z^{2}-2 z \overline{\lambda_{j}}+{\overline{\lambda_{j}}}^{2}+\overline{\lambda_{j}^{2}}-\bar{\lambda}_{j}^{2}\right) \\
& =j\left[\left(z-\overline{\lambda_{j}}\right)^{2}+\left(\overline{\lambda_{j}^{2}}-{\overline{\lambda_{j}}}^{2}\right)\right] \\
& \geq j\left(z-\overline{\lambda_{j}}\right)^{2} .
\end{aligned}
$$


Proof of Theorem 1.2 1) Substituting $z_{j}=3 \overline{\lambda_{j}}$ into (4.2) and noticing (4.3), we have

$$
R_{2}(z) \geq j\left(z_{j}-\overline{\lambda_{j}}\right)^{2} \frac{z^{3}}{z_{j}^{3}}=\frac{4 j z^{3}}{27 \overline{\lambda_{j}}}
$$

and (1.8) is proved.

2) We take (1.8) into (3.14) to obtain

$$
R_{1}(z) \geq \frac{3}{2 z} \cdot \frac{4 j z^{3}}{27 \overline{\lambda_{j}}}=\frac{2 j z^{2}}{9 \overline{\lambda_{j}}}
$$

and (1.9) is proved.

3) Combining (1.8) and (3.15), it implies

$$
N(z)=R_{0}(z) \geq \frac{9}{4 z^{2}} \cdot \frac{4 j z^{3}}{27 \overline{\lambda_{j}}}=\frac{j z}{3 \overline{\lambda_{j}}}
$$

and (1.10) is proved.

4) If $\lambda_{k+1} \leq 3 \overline{\lambda_{j}}$, then (1.11) is clearly valid; if $\lambda_{k+1}>3 \bar{\lambda}_{j}$, then (1.10) shows by letting $z \rightarrow \lambda_{k+1}$ that

$$
\frac{\lambda_{k+1}}{\lambda_{j}} \leq \frac{3 k}{j} \text {. }
$$

So (1.11) is proved and Theorem 1.2 is proved.

Corollary 4.1 We have

$$
\lambda_{k+1} \leq 3 \overline{\lambda_{k}}
$$

and

$$
\lambda_{k+1} \leq 3 k \lambda_{1} .
$$

\section{Proof of Theorem 1.3}

We first recall the following definition before proving Theorem 1.3.

Definition 5.1 If $f(z)$ is superlinear in $z$ as $z \rightarrow \infty$, then its Legendre transform is defined by

$$
L[f](w)=\sup _{z}\{w z-f(z)\} .
$$

Remark 5.2 If $f(z) \geq g(z)$ for all $z$, then $L[f](w) \leq L[g](w)$ for all $w$; Since the maximizing value of $z$ in (5.1) is a nondecreasing function of $w$, it follows that for $w$ sufficiently large, the maximizing $z$ exceeds $z_{j}=3 \overline{\lambda_{j}}$.

Proof of Theorem 1.3 From (1.9), we have

$$
L\left[R_{1}\right](w) \leq L\left[\frac{2 j z^{2}}{9 \bar{\lambda}_{j}}\right](w) .
$$

Now let us calculate $L\left[R_{1}\right](w)$. Since

$$
R_{1}(z)=\sum_{k}\left(z-\lambda_{k}\right)_{+}
$$

is piecewise linear function of $z$, it implies that the maximizing value of $z$ in the Legendre transform of $R_{1}$ is attained at one of the critical values.
In fact if $\lambda_{k}<z \leq \lambda_{k+1}$, then

$$
\begin{aligned}
L\left[R_{1}\right](w) & =\sup _{z}\left\{w z-R_{1}(z)\right\} \\
& =\sup _{z}\left\{w z-\sum_{k}\left(z-\lambda_{k}\right)_{+}\right\} \\
& =\sup _{z}\left\{w z-\left(z-\lambda_{1}\right)-\left(z-\lambda_{2}\right)-\cdots-\left(z-\lambda_{k}\right)\right\} \\
& =\sup _{z}\left\{(w-k) z+\lambda_{1}+\lambda_{2}+\cdots+\lambda_{k}\right\} .
\end{aligned}
$$

Noting that the maximizing value of $z$ is a nondecreasing function of $w$, we see $w-k \geq 0$, therefore the critical value $z_{c r}=\lambda_{k+1}$.

It is easy to check $k=[w]$ and

$$
\begin{aligned}
L\left[R_{1}\right](w) & =\sup _{z}\left\{(w-k) z+\lambda_{1}+\lambda_{2}+\cdots+\lambda_{k}\right\} \\
& =(w-[w]) \lambda_{[w]+1}+[w] \cdot \frac{\lambda_{1}+\lambda_{2}++\lambda_{[w]}}{[w]} \\
& =(w-[w]) \lambda_{[w]+1}+[w] \overline{\lambda_{[w]}},
\end{aligned}
$$

Next we calculate $L\left[\frac{2 j z^{2}}{9 \overline{\lambda_{j}}}\right](w)$. Noting

$$
L\left[\frac{2 j z^{2}}{9 \overline{\lambda_{j}}}\right](w)=\sup _{z}\left\{w z-\frac{2 j z^{2}}{9 \bar{\lambda}_{j}}\right\}
$$

and letting

$$
f(z)=w z-\frac{2 j z^{2}}{9 \overline{\lambda_{j}}},
$$

we know $f^{\prime}(z)=w-\frac{4 j z}{9 \overline{\lambda_{j}}}$. By $f^{\prime}(z)=0$, it solves

$$
Z_{*}=\frac{9 w \overline{\lambda_{j}}}{4 j} .
$$

Therefore

$$
\begin{aligned}
L\left[\frac{2 j z^{2}}{9 \overline{\lambda_{j}}}\right](w) & =\sup _{z}\left\{w z-\frac{2 j z^{2}}{9 \overline{\lambda_{j}}}\right\} \\
& =w \cdot \frac{9 w \overline{\lambda_{j}}}{4 j}-\frac{2 j}{9 \overline{\lambda_{j}}} \cdot\left(\frac{9 w \overline{\lambda_{j}}}{4 j}\right)^{2} \\
& =\frac{9 \overline{\lambda_{j}} w^{2}}{8 j} .
\end{aligned}
$$

Taking (5.3) and (5.5) into (5.2), we have

$$
(w-[w]) \lambda_{[w]+1}+[w] \overline{\lambda_{[w]}} \leq \frac{9 \overline{\lambda_{j}} w^{2}}{8 j},
$$

By (5.4), it has 


$$
w=\frac{4 j}{9 \bar{\lambda}_{j}} Z_{*}
$$

From Theorem 1.2, $Z_{*} \geq 3 \overline{\lambda_{j}}$, so $\quad w \geq \frac{4 j}{9 \bar{\lambda}_{j}} \cdot 3 \overline{\lambda_{j}}=\frac{4 j}{3}$.

Then it follows that if $w$ is restricted to the value $w \geq \frac{4 j}{3}$ then (5.6) is valid.

Meanwhile, for any $w$, we can always find an integer $k$ such that $k-1 \leq w<k$ and

$$
[w]=k-1 \text {. }
$$

If $k>\frac{4 j}{3}$ and $w$ approaches to $k$ from below, then we obtain from (5.5) that

$$
\lambda_{1}+\lambda_{2}+\cdots+\lambda_{k}=\lambda_{k}+(k-1) \overline{\lambda_{k-1}} \leq \frac{9 \overline{\lambda_{j}}}{8 j} k^{2} .
$$

Therefore

$$
\frac{\overline{\lambda_{k}}}{\overline{\lambda_{j}}} \leq \frac{9 k}{8 j}
$$

and Theorem 1.3 is proved.

Remark 5.3 If we let $j=1$, then

$$
\frac{\overline{\lambda_{k}}}{\lambda_{1}} \leq \frac{9}{8} k
$$

We point out that (5.7) is sharper than (4.4). In fact, we get from (4.4) that

$$
\sum_{j=0}^{k-1} \frac{\lambda_{j+1}}{\lambda_{1}} \leq 3 \sum_{j=0}^{k-1} j=\frac{3 k(k-1)}{2} \leq \frac{3}{2} k^{2}
$$

and

$$
\frac{\overline{\lambda_{k}}}{\lambda_{1}} \leq \frac{3}{2} k
$$

But $\frac{9 k}{8}<\frac{3 k}{2}$ is always valid, so (5.7) is sharper than (4.4).

\section{REFERENCES}

[1] N. Garofalo and F. Tournier, "New Properties of Convex Functions in the Heisenberg Group," Transactions of the
American Mathematical Society, Vol. 358, No. 5, 2005, pp. 2011-2055.

http://dx.doi.org/10.1090/S0002-9947-05-04016-X

[2] X. Luo and P. Niu, "Eigenvalues Problems for Square Sum Operators Consisting of Vector Fields," Mathematica Applicata, Vol. 10, No. 4, 1997, pp. 101-104.

[3] E. M. Harrell and L. Hermi, "On Riesz Means of Eigenvalues," Communications in Partial Differential Equations, Vol. 36, No. 9, 2011, pp. 1521-1543. http://dx.doi.org/10.1080/03605302.2011.595865

[4] Yu. G. Safarov, "Riesz Means of the Distribution Function of the Eigenvalues of an Elliptic Operator," Journal of Soviet Mathematics, Vol. 49, No. 5, 1990, pp. 12101212. http://dx.doi.org/10.1007/BF02208718

[5] B. Helffer and D. Robert, "Riesz Means of Bound States and Semicalassical Limit Connected with a Lieb-Thirring's Conjecture," Asymptotic Analysis, Vol. 3, No. 2, 1990, pp. 91-103.

[6] E. M. Harrell II and L. Hermi, "Differential Inequalities for Riesz Means and Weyl-Type Bounds for Eigenvalues," Journal of Functional Analysis, Vol. 254, No. 12, 2008, pp. 3171-3191. http://dx.doi.org/10.1016/j.jfa.2008.02.016

[7] G. Jia, J. Wang and Y. Xiong, "On Riesz Inequalities for Subelliptic Laplacian," Applied Mathematics, Vol. 2, No. 6, 2011, pp. 694-698. http://dx.doi.org/10.4236/am.2011.26091

[8] M. S. Ashbaugh and R. D. Benguria, "More Bounds on Eigenvalues Ratios for Dirichlet Laplacians in n Dimensions," SIAM Journal on Mathematical Analysis, Vol. 24, 1993, pp. 1622-1651. http://dx.doi.org/10.1137/0524091

[9] E. M. Harrell II and J. Stubbe, "On Trace Identities and Universal Eigenvalues Estimates for Some Partial Differential Operators," Transactions of the American Mathematical Society, Vol. 349, No. 5, 1997, pp pp. 17971809. http://dx.doi.org/10.1090/S0002-9947-97-01846-1

[10] P. Lvy-Bruhl, "Rsolubilit Locale et Global d'Opra-Teurs Invariants du Second Order sur des Group de Lie Nilpotents," Bulletin des Sciences Mathématiques, Vol. 104, No. 2, 1980, pp. 369-391.

[11] A. Laptev and T. Weidl, "Sharp Lieb-Thirring Inequalities in High Dimensions," Acta Mathematica, Vol. 184, No. 1, 2000, pp. 87-111. http://dx.doi.org/10.1007/BF02392782 